CUBO A Mathematical Journal

Vol.21, № 03, (39-61). December 2019

\title{
Stability And Boundedness In Nonlinear And Neutral Difference Equations using New Variation of Parameters Formula And Fixed Point Theory
}

\author{
Youssef N RAFFoul \\ Department of Mathematics, University of Dayton \\ Dayton, OH 45469-2316 USA \\ yraffoul1@udayton.edu
}

\begin{abstract}
In the case of nonlinear problems, whether in differential or difference equations, it is difficult and in some cases impossible to invert the problem and obtain a suitable mapping that can be effectively used in fixed point theory to qualitatively analyze its solutions. In this paper we consider the existence of a positive sequence and utilize it in the capacity of integrating factor to obtain a new variation of parameters formula. Then, we will use the obtained new variation of parameters formula and revert to the contraction principle to arrive at results concerning, boundedness, periodicity and stability. The author is working on parallel results for the continuous case.
\end{abstract}

\section{RESUMEN}

En el caso de problemas no-lineales, ya sea en ecuaciones diferenciales o en diferencias, es difícil y en algunos casos imposible invertir el problema y obtener una aplicación apropiada que pueda ser efectivamente usada en teoría de punto fijo para analizar quantitativamente sus soluciones. En este paper consideramos la existencia de una sucesión positiva y la usamos en la capacidad del factor de integración para obtener una nueva fórmula de variación de parámetros. Luego, usaremos esta nueva fórmula de variación de parámetros y volver al principio de contracción para obtener resultados que involucran acotamiento, periodicidad y estabilidad. El autor se encuentra trabajando en resultados paralelos para el caso continuo

Keywords and Phrases: New variation of parameters, Difference, Neutral, Sability, Boundedness, Fixed point theorems, Contraction mapping, equi-boundedness.

2010 AMS Mathematics Subject Classification: 39A11. 


\section{Introduction}

For motivational purpose we consider the linear difference equation

$$
x(t+1)=a(t) x(t), x\left(t_{0}\right)=x_{0}, t \geq t_{0} \geq 0 .
$$

It is clear that the solution of (1.1) is given by

$$
x(t)=x_{0} \prod_{s=t_{0}}^{t-1} a(s),
$$

provided that $a(t) \neq 0$ for all $t \in \mathbb{Z}^{+}$. Throughout this paper we adopt the convention that for any sequence $x(k)$

$$
\sum_{k=a}^{b} x(k)=0 \text { and } \prod_{k=a}^{b} x(k)=1 \text { whenever } a>b .
$$

For more on the calculus of difference equations we refer to [6]- [8] and [13].

Let $v(t)$ be a sequence such that $v: \mathbb{Z}^{+} \rightarrow \mathbb{R}$ with $v(t) \neq 0$ for all $t \in \mathbb{Z}^{+}$. Multiply both sides of (1.1) by $\prod_{s=t_{0}}^{t} v^{-1}(s)$ to obtain

$$
x(t+1) \prod_{s=t_{0}}^{t} v^{-1}(s)=a(t) x(t) \prod_{s=t_{0}}^{t} v^{-1}(s),
$$

Thus the above expression can be written in the compact form

$$
\Delta\left[x(t) \prod_{s=t_{0}}^{t-1} v^{-1}(s)\right]=[(a(t)-v(t)) x(t)] \prod_{s=t_{0}}^{t} a^{-1}(s) .
$$

Summing equation (1.3) from $t_{0}$ to t-1 gives

$$
x(t)=x_{0} \prod_{s=t_{0}}^{t-1} v(s)+\sum_{r=t_{0}}^{t-1}(a(r)-v(r)) x(r) \prod_{s=r+1}^{t-1} v(s) .
$$

Note that (1.4) reduces to (1.2) if we set $v(t)=a(t)$ in (1.4). To obtain asymptotic stability of the zero solution of (1.1) using (1.2) one would have to assume that

$$
\prod_{s=t_{0}}^{t} a(s) \rightarrow 0, \text { as } t \rightarrow \infty .
$$

On the hand, if we use (1.4) instead, then such requirement is not necessary. But instead, we would have to ask that

$$
\prod_{s=t_{0}}^{t} v(s) \rightarrow 0, \text { as } t \rightarrow \infty
$$


Such technique of inversion is of more importance when the right hand of (1.1) is either totally nonlinear or totally delayed. To see this, we consider the nonlinear difference equation

$$
x(t+1)=f(t, x(t)), x\left(t_{0}\right)=x_{0},
$$

where the function $f: \mathbb{Z}^{+} \times \mathbb{R} \rightarrow \mathbb{R}$ is continuous. The subject of stability and boundedness in difference equations is vast and we refer to [16] and [17]. We begin by stating some definitions .

Definition 1.1. We say $x(t):=x\left(t, t_{0}, x_{0}\right)$ is a solution of $(1.5)$ if $x\left(t_{0}\right)=x_{0}$ and satisfies (1.5) for $t \geq t_{0} \geq 0$.

Definition 1.2. The zero solution of (1.5) is stable if for any $\epsilon>0$ and any integer $t_{0} \geq 0$ there exists a $\delta>0$ such that $\left|x_{0}\right| \leq \delta$ implies $\left|x\left(t, t_{0}, x_{0}\right)\right| \leq \epsilon$ for $t \geq t_{0}$.

Definition 1.3. The zero solution of (1.5) is asymptotically stable if it is stable and $\left|x\left(t, t_{0}, x_{0}\right)\right| \rightarrow$ 0 as $t \rightarrow \infty$.

For more on stability we refer to [9] and [11]. We begin with the following Lemma. Its proof follows along the lines of the derivation of (1.4).

Lemma 1.4. If $x(t)$ is a solution of (1.5) on an interval $\mathbb{Z}^{+} \cap[0, T]$ and satisfies the initial condition $x\left(t_{0}\right)=x_{0}, t_{0} \geq 0$, then $x(t)$ is a solution of the summation equation if and only if

$$
x(t)=x_{0} \prod_{s=t_{0}}^{t-1} v(s)+\sum_{r=t_{0}}^{t-1}(f(r, x(r))-v(r) x(r)) \prod_{s=r+1}^{t-1} v(s),
$$

where $v: \mathbb{Z}^{+} \rightarrow \mathbb{R}$ with $v(t) \neq 0$ for all $t \in \mathbb{Z}^{+}$.

Next, we will use (1.6) to define a mapping on the proper space and show the zero solution is (AS). Let $\mathcal{C}$ be the set of all real-valued bounded sequences. Define the space

$$
\mathcal{S}=\{\phi:[0, \infty) \rightarrow \mathbb{R} / \phi \in \mathcal{C},|\phi(t)| \leq L, \phi(t) \rightarrow 0, \text { as } t \rightarrow \infty\}
$$

Then

$$
(\mathcal{S},\|\cdot\|)
$$

is a complete metric space under the uniform metric

$$
\rho\left(\phi_{1}, \phi_{2}\right)=\left\|\phi_{1}-\phi_{2}\right\|
$$

where

$$
\|\phi\|=\sup _{t \in \mathbb{Z}^{+}}\{|\phi(t)|\} .
$$

Assume

$$
f(t, 0)=0 .
$$


We assume the function $f$ is locally Lipschitz on the set $\mathcal{S}$.

That is, for any $\phi_{1}$ and $\phi_{2} \in \mathcal{S}$, we have

$$
\left|f\left(t, \phi_{1}\right)-f\left(t, \phi_{2}\right)\right| \leq \lambda(t)|| \phi_{1}-\phi_{2}||
$$

for $\lambda:[0, \infty) \rightarrow(0, \infty)$. Assume for $\phi \in \mathcal{S}$ and positve constant $L$, we have that

$$
\left|x_{0} \prod_{s=t_{0}}^{t-1} v(s)\right|+L \sum_{r=t_{0}}^{t-1}(|v(r)|+\lambda(r))\left|\prod_{s=r+1}^{t-1} v(s)\right| \leq L .
$$

Note that (1.9) implies that

$$
\sum_{r=t_{0}}^{t-1}(|v(r)|+\lambda(r))\left|\prod_{s=r+1}^{t-1} v(s)\right| \leq \alpha<1
$$

The next theorem offers results about stability and boundedness. For more results on the stability and boundedness using fixed point theory, we refer the interest reader to the book [18] and to the paper [19].

Theorem 1.5. Assume (1.7)-(1.9). Suppose there exists a positive constant $k$ such that

$$
\left|\prod_{s=t_{0}}^{t-1} v(s)\right| \leq k
$$

then the unique solution of (1.5) is bounded and its zero solution is stable.

If, in addition,

$$
\prod_{s=t_{0}}^{t-1} v(s) \rightarrow 0
$$

then the zero solution of (1.5) is asymptotically stable.

Proof. For $\phi \in \mathcal{S}$, define the mapping $\mathfrak{P}: \mathcal{S} \rightarrow \mathcal{S}$ by

$$
(\mathfrak{P} \phi)(t)=x_{0} \prod_{s=t_{0}}^{t-1} v(s)+\sum_{r=t_{0}}^{t-1}\left(f(r, \phi(r))-\phi(r) v(r) \prod_{s=r+1}^{t} v(s)\right.
$$

It is clear that $(\mathfrak{P} \phi)\left(t_{0}\right)=x_{0}$. Now for $\phi \in \mathcal{S}$, we have that

$$
|(\mathfrak{P} \phi)(t)| \leq\left|x_{0}\right| k+\sum_{r=t_{0}}^{t-1}(\lambda(r)|\phi(r)|+|\phi(r)| v(r))\left|\prod_{s=r+1}^{t} v(s)\right| .
$$

Consequently,

$$
\|\mathfrak{P} \phi\| \leq\left|x_{0}\right| k+\sum_{r=t_{0}}^{t-1}(|v(r)|+\lambda(r))\left|\prod_{s=r+1}^{t} v(s)\right|\|\phi\|
$$


Or,

$$
\|\mathfrak{P} \phi\| \leq\left|x_{0}\right| k+\alpha\|\phi\| \leq L .
$$

Since $\mathfrak{P}$ is continuous we have that $\mathfrak{P}: \mathcal{S} \rightarrow \mathcal{S}$. Next we show that $\mathfrak{P}$ is a contraction.

For $\phi_{1}, \phi_{2} \in \mathcal{S}$, we have from (1.12) that

$$
\begin{aligned}
\left|\left(\mathfrak{P} \phi_{1}\right)(t)-\left(\mathfrak{P} \phi_{2}\right)(t)\right| & \leq \sum_{r=t_{0}}^{t-1}(|v(r)|+\lambda(r))\left|\prod_{s=r+1}^{t} v(s)\right||| \phi_{1}-\phi_{2} \| \\
& \leq \alpha\left\|\phi_{1}-\phi_{2}\right\| .
\end{aligned}
$$

This shows that $\mathfrak{P}$ is a contraction. By Banach's contraction mapping principle, $\mathfrak{P}$ has a unique fixed point $x \in \mathcal{S}$ which is bounded. Moreover, the unique fixed point is a solution of (1.5) on $[0, \infty)$. Next we show the zero solution is stable. Let $x$ be the unique solution. Let $\varepsilon>0$ be given and chose $\delta=\varepsilon \frac{1-\alpha}{k}$. Thus for $\left|x_{0}\right|<\delta$, we have by (1.13) that

$$
(1-\alpha)|| x|| \leq\left|x_{0}\right| k<\delta k
$$

Or

$$
\|x\| \leq \varepsilon
$$

Left to prove that

$$
(\mathfrak{P} \varphi)(\mathfrak{t}) \rightarrow 0, \text { as } t \rightarrow \infty .
$$

We have already proved that the zero solution of (1.5) is stable. Let $\delta$ be the one from stability such that $\left|x_{0}\right|<\delta$ and define

$$
\mathcal{S}^{*}=\left\{\varphi: \mathbb{Z}^{+} \rightarrow \mathbb{R} \mid \varphi\left(t_{0}\right)=x_{0},\|\varphi\| \leq \epsilon \text { and } \varphi(t) \rightarrow 0 \text { as } t \rightarrow \infty\right\} .
$$

Let $\mathfrak{P}$ be given by (1.12) and define $\mathfrak{P}: S^{*} \rightarrow S^{*}$. The map $\mathfrak{P}$ is contraction and it maps from $S^{*}$ into itself.

We next show that $(\mathfrak{P} \varphi)(t)$ goes to zero as t goes to infinity.

The first term on the right of (1.12) goes to zero due to condition (1.11). Left to show that

$$
\left|\sum_{r=t_{0}}^{t-1}(f(r, x(r))-v(r) \phi(r)) \prod_{s=r+1}^{t-1} v(s)\right| \rightarrow 0, \text { as } t \rightarrow \infty .
$$

Let $\varphi \in S^{*}$ then $|\varphi(t)| \leq \epsilon$. Also, since $\varphi(t) \rightarrow 0$ as $t \rightarrow \infty$, there exists a $t_{1}>0$ such that for $t>t_{1},|\varphi(t)|<\epsilon_{1}$ for $\epsilon_{1}>0$. Due to condition (1.11) there exists a $t_{2}>t_{1}$ such that for $t>t_{2}$ implies that

$$
\left|\prod_{s=t_{1}}^{t} v(s)\right|<\frac{\epsilon_{1}}{\alpha \epsilon}
$$


Thus for $t>t_{2}$, we have

$$
\begin{aligned}
& \left|\sum_{r=t_{0}}^{t-1}(f(r, x(r))-v(r) \phi(r)) \prod_{s=r+1}^{t-1} v(s)\right| \leq \sum_{r=t_{0}}^{t-1}(\lambda(r)+v(r))|\phi(r)|\left|\prod_{s=r+1}^{t-1} v(s)\right| \\
\leq & \sum_{r=t_{0}}^{t_{1}-1}(\lambda(r)+v(r))|\phi(r)| \prod_{s=r+1}^{t-1} v(s) \mid \\
& +\sum_{r=t_{1}}^{t-1}(\lambda(r)+v(r))|\phi(r)|\left|\prod_{s=r+1}^{t-1} v(s)\right| \\
\leq & \epsilon \sum_{r=t_{0}}^{t_{1}-1}(\lambda(r)+v(r))|\phi(r)|\left|\prod_{s=r+1}^{t-1} v(s)\right|+\epsilon_{1} \alpha \\
\leq & \epsilon \sum_{r=t_{0}}^{t_{1}-1}(\lambda(r)+v(r))\left|\prod_{s=r+1}^{t_{1}-1} v(s)\right| \prod_{s=t_{1}}^{t-1} v(s) \mid+\epsilon_{1} \alpha \\
\leq & \epsilon\left|\prod_{s=t_{1}}^{t-1} v(s)\right| \sum_{r=t_{0}}^{t_{1}-1}(\lambda(r)+v(r))\left|\prod_{s=r+1}^{t_{1}-1} v(s)\right|+\epsilon_{1} \alpha \\
\leq & \epsilon \alpha\left|\prod_{s=t_{1}}^{t-1} v(s)\right|+\epsilon_{1} \alpha \\
\leq & \epsilon_{1}+\epsilon_{1} \alpha .
\end{aligned}
$$

Since $\epsilon_{1}$ is arbitrary small, this shows that $(\mathfrak{P} \varphi)(t) \rightarrow 0$ as $t \rightarrow \infty$. As $\mathfrak{P}$ has a unique fixed point, say $x$ it implies the asymptotic stability of the zero solution of (1.11). This completes the proof.

\section{Contraction Versus Large Contraction}

Now we consider particular nonlinear equation and rewrite so we can invert the usual way. Consequently, contraction mapping principle can no longer be useful. Let

$$
f(t, x)=-a(t) x^{3}+l(t, x),
$$

where $l(t, x)$ continuous and satisfies a smallness condition. Thus, We consider

$$
x(t+1)=-a(t) x^{3}+l(t, x) .
$$

mentioned paper as an example for illustrating the need for Large Contraction. In [4], the author put (1.12) in the form

$$
x(t+1)=-a(t) x+a(t)\left(x-x^{3}\right)+l(t, x) .
$$


Then by the variation of parameters formula we have

$$
x(t)=x_{0} \prod_{s=t_{0}}^{t-1} a(s)+\sum_{r=t_{0}}^{t-1}\left(a(r)\left(x(r)-x^{3}(r)\right)+l(t, x(r))\right) \prod_{s=r+1}^{t-1} a(s) .
$$

It is naive to believe that every map can be defined so that it is a contraction, even with the strictest conditions. To see this, we consider

$$
g(x)=x-x^{3}
$$

then for $x, y \in \mathbb{R}$ with $|x|,|y| \leq \frac{\sqrt{3}}{3}$ we have that

$$
|g(x)-g(y)|=\left|x-x^{3}-y+y^{3}\right| \leq|x-y|\left(1-\frac{x^{2}+y^{2}}{2}\right)
$$

and the contraction constant tends to one as $x^{2}+y^{2} \rightarrow 0$. As a consequence, the regular contraction mapping principle failed to produce any results. For more on this and Large contraction, we refer to [18], P: 52. To get around it, we let $v(t)$ be a sequence such that $v: \mathbb{Z}^{+} \rightarrow \mathbb{R}$ with $v(t) \neq 0$ for all $t \in \mathbb{Z}^{+}$. By similar steps as in the development of (1.4) we arrive at the variation of parameters formula

$$
x(t)=x_{0} \prod_{s=t_{0}}^{t-1} v(s)+\sum_{r=t_{0}}^{t-1}\left(v(r) x(r)-a(t) x^{3}(r)+l(t, x(r))\right) \prod_{s=r+1}^{t-1} v(s)
$$

Thus, one can show that the map given by

$$
f(x)=v(r) x(r)-a(t) x^{3}(r),
$$

is a contraction on some bounded and small set provided $a$ and $v$ have small magnitudes. To better illustrate our intention we set $l(t, x)=0$, and consider (2.1). Then from the above variation of parameters formula, we have that

$$
\left.x(t)=x_{0} \prod_{s=t_{0}}^{t-1} v(s)+\sum_{r=t_{0}}^{t-1}\left(v(r) x(r)-a(t) x^{3}(r)\right)\right) \prod_{s=r+1}^{t-1} v(s) .
$$

Assume for $\phi \in \mathcal{S}$ and positve constant $L$, we have that

$$
\left|x_{0} \prod_{s=t_{0}}^{t-1} v(s)\right|+\sum_{r=t_{0}}^{t-1}\left(L|v(r)|+L^{3}|a(r)|\right)\left|\prod_{s=r+1}^{t-1} v(s)\right| \leq L,
$$

and

$$
\sum_{r=t_{0}}^{t-1}\left(|v(r)|+3 L^{2}|a(r)|\right)\left|\prod_{s=r+1}^{t-1} v(s)\right| \leq \alpha<1 .
$$

The next theorem offers results about stability and boundedness. For more results on the stability and boundedness using fixed point theory, we refer the interest reader to the book [18] and to the paper [19]. 
Theorem 2.1. Assume (1.7), (1.10), (2.6) and (2.7). Then the unique solution of (2.1) is bounded and its zero solution is stable.

If, in addition, (1.11) holds, then the zero solution of (2.1) is asymptotically stable.

Proof. For $\phi \in \mathcal{S}$, define the mapping $\mathfrak{P}: \mathcal{S} \rightarrow \mathcal{S}$, by

$$
(\mathfrak{P} \phi)(t)=x_{0} \prod_{s=t_{0}}^{t-1} v(s)+\sum_{r=t_{0}}^{t-1}\left(v(r) \phi(r)-\phi^{3}(r) a(r)\right) \prod_{s=r+1}^{t} v(s)
$$

It is clear that $(\mathfrak{P} \phi)\left(t_{0}\right)=x_{0}$. Now for $\phi \in \mathcal{S}$, we have that

$$
\begin{aligned}
|(\mathfrak{P} \phi)(t)| & \leq\left|x_{0}\right| k+\sum_{r=t_{0}}^{t-1}\left(|v(r)||\phi(r)|+\left|\phi^{3}(r)\right||a(r)|\right)\left|\prod_{s=r+1}^{t} v(s)\right| \\
& \leq\left|x_{0} \prod_{s=t_{0}}^{t-1} v(s)\right|+\sum_{r=t_{0}}^{t-1}\left(L|v(r)|+L^{3}|a(r)|\right)\left|\prod_{s=r+1}^{t-1} v(s)\right| .
\end{aligned}
$$

Thus,

$$
\|\mathfrak{P} \phi\| \leq L .
$$

Since $\mathfrak{P}$ is continuous we have that $\mathfrak{P}: \mathcal{S} \rightarrow \mathcal{S}$. Next we show that $\mathfrak{P}$ is a contraction.

For $\phi_{1}, \phi_{2} \in \mathcal{S}$, we have from (1.12) that

$$
\begin{aligned}
\left|\left(\mathfrak{P} \phi_{1}\right)(t)-\left(\mathfrak{P} \phi_{2}\right)(t)\right| & \leq \sum_{r=t_{0}}^{t-1}\left(|v(r)|\left|\phi_{1}(r)-\phi_{2}(r)\right|\right. \\
& +\sum_{r=t_{0}}^{t-1}|a(r)|\left|\phi_{1}(r)-\phi_{2}(r)\right|\left(\phi_{1}^{2}(r)+\left|\phi_{1}(r) \phi_{2}(r)\right|+\phi_{1}^{2}(r)\right)\left|\prod_{s=r+1}^{t} v(s)\right| \\
& \leq \sum_{r=t_{0}}^{t-1}\left(|v(r)|+3 L^{2}|a(r)|\right)\left|\prod_{s=r+1}^{t-1} v(s)\right||| \phi_{1}-\phi_{2}|| \\
& \leq \alpha\left\|\phi_{1}-\phi_{2}\right\| .
\end{aligned}
$$

This shows that $\mathfrak{P}$ is a contraction. By Banach's contraction mapping principle, $\mathfrak{P}$ has a unique fixed point $x \in \mathcal{S}$ which is bounded. The proof for stability and asymptotic stability follow along the lines of the proof of Theorem 1.5.

For the rest of this section we set $l(t, x)=0$ in (2.3) and use large contraction and prove parallel theorem to Theorem 2.1. We saw before that the function or map, $g(x)=x-x^{3}$ does not define a contraction. To get around it we use the notion of large contraction that was introduced by Burton in [5]. We will restate the contraction mapping principle and Krasnoselskii's fixed point theorems in which the regular contraction is replaced with large contraction. Then based on the notion of large contraction, we introduce a theorem to obtain boundedness results in which large contraction is substituted for regular contraction. 
Definition 2.2. Let $(\mathcal{M}, d)$ be a metric space and $B: \mathcal{M} \rightarrow \mathcal{M}$. The map $B$ is said to be large contraction if $\phi, \varphi \in \mathcal{M}$, with $\phi \neq \varphi$ then $d(B \phi, B \varphi) \leq d(\phi, \varphi)$ and if for all $\varepsilon>0$, there exists a $\delta \in(0,1)$ such that

$$
[\phi, \varphi \in \mathcal{M}, d(\phi, \varphi) \geq \varepsilon] \Rightarrow d(B \phi, B \varphi) \leq \delta d(\phi, \varphi)
$$

The next theorems are alternative to the regular contraction mapping principle and Krasnoselskii's fixed point theorem in which we substitute Large Contraction for regular contraction. The proofs of the two theorems and the statement of Definition 2.2 can be found in [5].

Theorem 2.3. Let $(\mathcal{M}, \rho)$ be a complete metric space and $B$ be a large contraction. Suppose there are an $x \in \mathcal{M}$ and an $L>0$ such that $\rho\left(x, B^{n} x\right) \leq L$ for all $n \geq 1$. Then $B$ has a unique fixed point in $\mathcal{M}$.

Next we state and prove a remarkable theorem by Adivar, Raffoul and Islam that generalizes the concept of Large Contraction. Its proof can be found in [18]. The theorem provides easily checked sufficient conditions under which a mapping is a large contraction. Several authors have published it in their work without the proper citations.

Consider the mapping $H$ defined by

$$
H(x(u))=x(u)-h(x(u))
$$

Let $\alpha \in(0,1]$ be a fixed real number and define the set $\mathbb{M}_{\alpha}$ by

$$
\mathbb{M}_{\alpha}=\{\phi: \phi \in C(\mathbb{R}, \mathbb{R}) \text { and }\|\phi\| \leq \alpha\}
$$

H.1. $h: \mathbb{R} \rightarrow \mathbb{R}$ is continuous on $[-\alpha, \alpha]$ and differentiable on $(-\alpha, \alpha)$,

H.2. The function $h$ is strictly increasing on $[-\alpha, \alpha]$,

$$
\text { H.3. } \sup _{t \in(-\alpha, \alpha)} h^{\prime}(t) \leq 1 \text {. }
$$

Theorem 2.4. ([1] )[Adivar-Raffoul-Islam] (Classifications of Large Contraction Theorem) Let $h: \mathbb{R} \rightarrow \mathbb{R}$ be a function satisfying (H.1-H.3). Then the mapping $H$ in (2.9) is a large contraction on the set $\mathbb{M}_{\alpha}$.

Example 2.5. Let $\alpha \in(0,1)$ and $k \in \mathbb{N}$ be fixed elements and $u \in(-1,1)$.

1. The condition (H.2) is not satisfied for the function $h_{1}(u)=\frac{1}{2 k} u^{2 k}$.

2. The function $h_{2}(u)=\frac{1}{2 k+1} u^{2 k+1}$ satisfies (H.1-H.3).

Proof. Since $h_{1}^{\prime}(u)=u^{2 k-1}<0$ for $-1<u<0$, the condition (H.2) is not satisfied for $h_{1}$. Evidently, (H.1-H.2) hold for $h_{2}$. (H.3) follows from the fact that $h_{2}^{\prime}(u) \leq \alpha^{2 k}$ and $\alpha \in(0,1)$. 
We have the following lemma. Define the mapping

$$
H(x)=x-x^{3} .
$$

Lemma 2.6. Let $\|\cdot\|$ denote the supremum norm. If

$$
\mathbb{M}=\left\{\phi: \mathbb{Z} \rightarrow \mathbb{R} \mid \phi(0)=\phi_{0}, \quad \text { and }\|\phi\| \leq \frac{\sqrt{3}}{3}\right\},
$$

then the mapping $H$ defined by (2.11) is a large contraction on the set $\mathbb{M}$.

Proof. Let $\alpha=\frac{\sqrt{3}}{3}$ and $h(x)=x^{3}$. Then, clearly $h$ satisfies (H.1-H.2). Moreover, $\sup _{x \in(-\alpha, \alpha)} h^{\prime}(x)=1$, which satisfies H.3. Hence by Theorem 2.4 defines a large contraction.

For $\psi \in \mathbb{M}$, we define the map $B: \mathbb{M} \rightarrow \mathbb{M}$ by

$$
(B \psi)(t)=\psi_{0} \prod_{s=0}^{t-1} a(s)+\sum_{s=0}^{t-1}\left(a(s) H(\psi(s)) \prod_{u=s+1}^{t-1} a(u)\right)
$$

Lemma 2.7. Assume for all $t \in \mathbb{Z}$

$$
\left|\psi_{0}\right|\left|\prod_{s=0}^{t-1} a(s)\right|+\frac{2 \sqrt{3}}{9} \sum_{s=0}^{t-1}\left|\prod_{u=s}^{t-1} a(u)\right| \leq \frac{\sqrt{3}}{3} .
$$

If $H$ is a large contraction on $\mathbb{M}$, then so is the mapping $B$.

Proof. It is easy to see that

$$
|H(x(t))|=\left|x(t)-x(t)^{3}\right| \leq \frac{2 \sqrt{3}}{9} \text { for all } x \in \mathbb{M} .
$$

By Lemma 2.6, $H$ is a large contraction on $\mathbb{M}$. Hence, for $x, y \in \mathbb{M}$ with $x \neq y$, we have $\|H x-H y\| \leq$ $\|x-y\|$. Hence,

$$
\begin{aligned}
|B x(t)-B y(t)| & \leq \sum_{s=0}^{t-1}|H(x(s))-H(y(s))|\left|\prod_{u=s}^{t-1} a(u)\right| \\
& \leq \frac{2 \sqrt{3}}{9} \sum_{s=0}^{t-1}\left|\prod_{u=s}^{t-1} a(u)\right|\|x-y\| \\
& =\|x-y\| .
\end{aligned}
$$

Taking supremum norm over the set $[0, \infty)$, we get that $\|B x-B y\| \leq\|x-y\|$. For a given $\varepsilon \in(0,1)$, suppose $x, y \in \mathbb{M}$ with $\|x-y\| \geq \varepsilon$. Then for $\delta=\min \left\{1-\varepsilon^{2} / 16,1 / 2\right\}$, which implies that $0<\delta<1$. Hence, for all such $\varepsilon>0$ we know that

$$
[x, y \in \mathbb{M},\|x-y\| \geq \varepsilon] \Rightarrow\|H x-H y\| \leq \delta\|x-y\| .
$$


Therefore, using (2.13), one easily verify that

$$
\|B x-B y\| \leq \delta\|x-y\| .
$$

The proof is complete.

We arrive at the following theorem in which we prove boundedness.

Theorem 2.8. Assume (2.13). Then (2.1) has a unique solution in $\mathbb{M}$ which is bounded.

Proof. ( $\mathbb{M},\|\cdot\|)$ is a complete metric space of bounded sequences. For $\psi \in \mathbb{M}$ we must show that $(B \psi)(t) \in \mathbb{M}$. From $(2.12)$ and the fact that

$$
|H(x(t))|=\left|x(t)-x(t)^{3}\right| \leq \frac{2 \sqrt{3}}{9} \text { for all } x \in \mathbb{M},
$$

we have

$$
\begin{aligned}
|(B \psi)(t)| & \leq\left|\psi_{0}\right|\left|\prod_{s=0}^{t-1} a(s)\right|+\frac{2 \sqrt{3}}{9} \sum_{s=0}^{t-1}\left|\prod_{u=s}^{t-1} a(u)\right| \\
& \leq \frac{\sqrt{3}}{3} .
\end{aligned}
$$

This shows that $(B \psi)(t) \in \mathbb{M}$. Lemma 2.6 implies the map $B$ is a large contraction and hence by Theorem 2.3, the map $B$ has a unique fixed point in $\mathbb{M}$ which is a solution of (2.1). This completes the proof.

\section{Periodic Solutions}

In this section we apply our new method to linear or nonlinear difference equations to show the existence of periodic solutions without the requirement of some classic conditions. To better illustrate our approach, we consider the nonlinear difference equation

$$
x(t+1)=a(t) x(t)+f(t, x(t))
$$

where $f$ is continuous in $x$. Let $\mathrm{T}$ be an integer such that $T \geq 1$. We assume the periodicity condition

$$
a(t+T)=a(t), \text { and } f(t+T, \cdot)=f(t, \cdot) .
$$

Let $B C$ is the space of bounded sequences $\phi: \mathbb{Z} \rightarrow \mathbb{R}$ with the maximum norm $\|\cdot\|$. Define $P_{T}=\{\phi \in B C, \phi(t+T)=\phi(t)\}$. Then $P_{T}$ is a Banach space when it is endowed with the maximum norm

$$
\|x\|=\max _{t \in[0, T-1]}|x(t)| .
$$


Also, we assume that

$$
\prod_{s=t-T}^{t-1} a(s) \neq 1
$$

Throughout this section we assume that $a(t) \neq 0$ for all $t \in[0, T-1]$. Let $x \in P_{T}$. Then Eqn. (3.1) is equivalent to

$$
\Delta\left[x(t) \prod_{s=t_{0}}^{t-1} a^{-1}(s)\right]=f(t, x(t)) \prod_{s=t_{0}}^{t} a^{-1}(s) .
$$

Summing equation (3.4) from $t-T$ to $t-1$ and using the fact that $x(t-T)=x(t)$, gives

$$
x(t)=\left(1-\prod_{s=t-T}^{t-1} a(s)\right)^{-1} \sum_{r=t-T}^{t-1} f(r, x(r)) \prod_{s=r+1}^{t-1} a(s) .
$$

Define the mapping $\mathfrak{P}$ on $P_{T}$ by

$$
(\mathfrak{P} \phi)(t)=\left(1-\prod_{s=t-T}^{t-1} a(s)\right)^{-1} \sum_{r=t-T}^{t-1} f(r, \phi(r)) \prod_{s=r+1}^{t-1} a(s) .
$$

One can easily verify that $(\mathfrak{P} \phi)(t+T)=(\mathfrak{P} \phi)(t)$, and hence $\mathfrak{P}: P_{T} \rightarrow P_{T}$.

Theorem 3.1. Suppose $a(t) \neq 0$ for all $t \in[0, T-1]$ and assume (3.3). Suppose the function $f$ is Lipschitz continuous with Lipschitz constant $k$. If

$$
k\left|\left(1-\prod_{s=t-T}^{t-1} a(s)\right)^{-1}\right| \sum_{r=t-T}^{t-1}\left|\prod_{s=r+1}^{t-1} a(s)\right| \leq \alpha,
$$

for $\alpha \in(0,1)$, then Eqn. (3.1) has a unique periodic solution.

Proof. The proof is easily obtained by direct application of contraction mapping principle on the set $P_{T}$.

Next, we use our new technique to avoid the requirement that $a(t) \neq 0$ for all $t \in[0, T-1]$ along with condition (3.3). Let $v(t)$ be a sequence such that $v: \mathbb{Z}^{+} \rightarrow \mathbb{R}$ with $v(t) \neq 0$ for all $t \in\left[0, T-1\right.$. Assume (3.2) and for $v \in P_{T}$, multiply both sides of $(3.1)$ by $\prod_{s=t_{0}}^{t} v^{-1}(s)$ to obtain

$$
\Delta\left[x(t) \prod_{s=t_{0}}^{t-1} v^{-1}(s)\right]=\left[(a(t) x(t)-v(t) x(t)+f(t, x(t))] \prod_{s=t_{0}}^{t} v^{-1}(s) .\right.
$$

Summing equation (3.7) from $t-T$ to $t-1$ gives and using the fact that $x(t-T)=x(t)$, gives

$$
x(t)=\left(1-\prod_{s=t-T}^{t-1} v(s)\right)^{-1} \sum_{r=t-T}^{t-1}[a(r) x(r)-v(r) x(r)+f(r, x(r))] \prod_{s=r+1}^{t-1} v(s) .
$$


Define the mapping $\mathfrak{P}$ on $P_{T}$ by

$$
(\mathfrak{P} \phi)(t)=\left(1-\prod_{s=t-T}^{t-1} v(s)\right)^{-1} \sum_{r=t-T}^{t-1}\left[a(r) x(r)-v(r) x(r)+f(r, \phi(r)) \prod_{s=r+1}^{t-1} v(s) .\right.
$$

One can easily verify that $(\mathfrak{P} \phi)(t+T)=(\mathfrak{P} \phi)(t)$, and hence $\mathfrak{P}: P_{T} \rightarrow P_{T}$.

Theorem 3.2. Suppose $v(t) \neq 0$ for all $t \in[0, T-1]$ and assume

$$
\prod_{s=t-T}^{t-1} v(s) \neq 1
$$

Suppose the function $f$ is Lipschitz continuous with Lipschitz constant $k$. If

$$
\left|\left(1-\prod_{s=t-T}^{t-1} v(s)\right)^{-1}\right| \sum_{r=t-T}^{t-1}[|a(r)|+|v(r)|+k]\left|\prod_{s=r+1}^{t-1} v(s)\right| \leq \alpha,
$$

for $\alpha \in(0,1)$, then Eqn. (3.1) has a unique periodic solution.

Proof. The proof is easily obtained by direct application of the contraction mapping principle on the set $P_{T}$.

Next we display an example.

Example 3.3. For positive constant $k$, we consider the difference equation

$$
x(t+1)=\left(1-(-1)^{t}\right) x(t)+\frac{k x}{1+x^{2}}
$$

It is clear that $a(t)=\left(1-(-1)^{t}\right)$ is periodic of period $T=2$ and $a(0)=0$. Hence Theorem 3.1 can not be applied. On the other hand we may apply Theorem 3.2 by taking $v(t)=\frac{(-1)^{t}}{2}$, for sufficiently small $k$.

\section{Neutral Difference Equations}

We extend the results of the previous sections to the neutral difference equation with functional delay

$$
x(t+1)=a(t) x(t)+b(t) x(t-g(t))+c(t) \Delta x(t-g(t))
$$

where where $a, b, c: \mathbb{Z} \rightarrow \mathbb{R}$, and $g: \mathbb{Z} \rightarrow \mathbb{Z}^{+}$. Moreover, we will discuss the concept of equiboundedness.

If for some positive constant $k,|g| \leq k$ then for any integer $t_{0} \geq 0$, we define $\mathbb{Z}_{0}$ to be the set of integers in $\left[t_{0}-k, t_{0}\right]$. If $g$ is unbounded then $\mathbb{Z}_{0}$ will be the set of integers in $\left(-\infty, t_{0}\right]$. We assume 
the existence of a given bounded initial sequence $\psi(t): \mathbb{Z}_{0} \rightarrow \mathbb{R}$. We will use the summation by parts formula

$$
\sum(E x(t) \Delta z(t))=x(t) z(t)-\sum z(t) \Delta x(t)
$$

where $E$ is defined as $E x(t)=x(t+1)$.

Definition 4.1. We say $x(t):=x\left(t, t_{0}, \psi\right)$ is a solution of (4.1) if $x(t)=\psi(t)$ on $\mathbb{Z}_{0}$ and satisfies (4.1) for $t \geq t_{0}$.

Definition 4.2. The zero solution of (4.1) is stable if for any $\epsilon>0$ and any integer $t_{0} \geq 0$ there exists a $\delta>0$ such that $|\psi(t)| \leq \delta$ on $\mathbb{Z}_{0}$ implies $\left|x\left(t, t_{0}, \psi\right)\right| \leq \epsilon$ for $t \geq t_{0}$.

Definition 4.3. The zero solution of (4.1) is asymptotically stable if it is stable and if for any integer $t_{0} \geq 0$ there exists $r\left(t_{0}\right)>0$ such that $|\psi(t)| \leq r\left(t_{0}\right)$ on $\mathbb{Z}_{0}$ implies $\left|x\left(t, t_{0}, \psi\right)\right| \rightarrow 0$ as $t \rightarrow$ $\infty$.

Definition 4.4. A solution $x\left(t, t_{0}, \psi\right)$ of $(4.1)$ is said to be bounded if there exist a $B\left(t_{0}, \psi\right)>0$ such that $\left|x\left(t, t_{0}, \psi\right)\right| \leq B\left(t_{0}, \psi\right)$ for $t \geq t_{0}$.

Definition 4.5. The solutions of (4.1) are said to be equi-bounded if for any $t_{0}$ and any $B_{1}>0$, there exists a $B_{2}=B_{2}\left(t_{0}, B_{1}\right)>0$ such that $|\psi(t)| \leq B_{1}$ on $\mathbb{Z}_{0}$ implies $\left|x\left(t, t_{0}, \psi\right)\right| \leq B_{2}$ for $t \geq t_{0}$.

For the remaining of the section we assume that there is a positive constant $k,|g| \leq k$.

Lemma 4.6. If $x(t)$ is a solution of (4.1) and satisfies the initial condition $x(t)=\psi(t)$ for $t \in \mathbb{Z}_{0}$, then $x(t)$ is a solution of the summation equation if and only if

$$
\begin{aligned}
x(t) & =\left[x\left(t_{0}\right)-c\left(t_{0}-1\right) x\left(t_{0}-g\left(t_{0}\right)\right)\right] \prod_{s=t_{0}}^{t-1} v(s)+c(t-1) x(t-g(t)) \\
& +\sum_{r=t_{0}}^{t-1}\left[(a(r)-v(r)) x(r) \prod_{s=r+1}^{t-1} v(s)\right] \\
& +\sum_{r=t_{0}}^{t-1}\left([b(r)-\phi(r)] x(r-g(r)) \prod_{s=r+1}^{t-1} v(s)\right), t \geq t_{0}
\end{aligned}
$$

where

$$
\phi(r)=c(r)-c(r-1) v(r) .
$$

$[0, T]$ where $v: \mathbb{Z} \cap[-k, \infty) \rightarrow \mathbb{R}$ with $v(t) \neq 0$.

Multiply both sides of (4.1) by $\prod_{s=t_{0}}^{t} v^{-1}(s)$ and then notice the resulting expression is equivalent 
to

$$
\begin{aligned}
\Delta\left[x(t) \prod_{s=t_{0}}^{t-1} v^{-1}(s)\right] & =[(a(t)-v(t)) x(t)+b(t) x(t-g(t)) \\
& +c(t) \Delta x(t-g(t))] \prod_{s=t_{0}}^{t} v^{-1}(s)
\end{aligned}
$$

Summing the above expression from $t_{0}$ to $\mathrm{t}-1$ gives

$$
\begin{aligned}
x(t) \prod_{s=t_{0}}^{t-1} v^{-1}(s)-x\left(t_{0}\right) & =\sum_{r=t_{0}}^{t-1}[(a(r)-v(r)) x(r) \\
& +b(r) x(r-g(r))+c(r) \Delta x(r-g(r))] \prod_{s=t_{0}}^{r} v^{-1}(s)
\end{aligned}
$$

Dividing both sides by $\prod_{s=t_{0}}^{t-1} v^{-1}(s)$, gives

$$
\begin{aligned}
x(t)= & x\left(t_{0}\right) \prod_{s=t_{0}}^{t-1} v(s)+\sum_{r=t_{0}}^{t-1}\left[(a(r)-v(r)) x(r) \prod_{s=r+1}^{t-1} v(s)\right] \\
+ & \sum_{r=t_{0}}^{t-1}[b(r) x(r-g(r)) \\
& +c(r) \Delta x(r-g(r))] \prod_{s=t_{0}}^{r} v^{-1}(s) \prod_{s=t_{0}}^{t-1} v(s) \\
& =x\left(t_{0}\right) \prod_{s=t_{0}}^{t-1} v(s)+\sum_{r=t_{0}}^{t-1}\left[(a(r)-v(r)) x(r) \prod_{s=r+1}^{t-1} v(s)\right] \\
+ & \sum_{r=t_{0}}^{t-1}[b(r) x(r-g(r))] \prod_{s=r+1}^{t-1} v(s) \\
& +\sum_{r=t_{0}}^{t-1}[c(r) \Delta x(r-g(r))] \prod_{s=r+1}^{t-1} v(s)
\end{aligned}
$$

Using summation by parts and after some calculations and simplification we arrive at (4.2).

Theorem 4.7. Suppose $v(t) \neq 0$ for $t \geq t_{0}$ and $v(t)$ satisfies

$$
\left|\prod_{s=t_{0}}^{t-1} v(s)\right| \leq M
$$


for $M>0$. Also, suppose that there is an $\alpha \in(0,1)$ such that

$$
\begin{aligned}
|c(t-1)| & +\sum_{r=t_{0}}^{t-1}|a(r)-v(r)| \prod_{s=r+1}^{t-1} v(s) \mid \\
& +\sum_{r=t_{0}}^{t-1}[|b(r)-\phi(r)|]\left|\prod_{s=r+1}^{t-1} a(s)\right| \leq \alpha, t \geq t_{0} .
\end{aligned}
$$

Then solutions of (4.1) are equi-bounded.

Proof. Let $B_{1}$ and $B_{2}$ be two positive constants to be defined later in the proof and let $\psi(t)$ be a bounded initial function satisfying $|\psi(t)| \leq B_{1}$ on $\mathbb{Z}_{0}$. Define

$$
S=\left\{\varphi: \mathbb{Z} \rightarrow \mathbb{R} \mid \varphi(t)=\psi(t) \text { on } \mathbb{Z}_{0} \text { and }\|\varphi\| \leq B_{2}\right\},
$$

where

$$
\begin{gathered}
\|\varphi\|=\sup _{t \in \mathbb{Z}}|\varphi(t)| .
\end{gathered}
$$

Then $(S,\|\cdot\|)$ is a complete metric space.

Define mapping $P: S \rightarrow S$ by

$$
(P \varphi)(t)=\psi(t) \text { on } \mathbb{Z}_{0}
$$

and

$$
\begin{aligned}
(P \varphi)(t)= & {\left[\psi\left(t_{0}\right)-c\left(t_{0}-1\right) \psi\left(t_{0}-g\left(t_{0}\right)\right)\right] \prod_{s=t_{0}}^{t-1} v(s)+c(t-1) \varphi(t-g(t)) } \\
& +\sum_{r=t_{0}}^{t-1}\left[(a(r)-v(r)) \varphi(r) \prod_{s=r+1}^{t-1} v(s)\right] \\
& +\sum_{r=t_{0}}^{t-1}\left[(b(r)-\phi(r)) \varphi(r-g(r)) \prod_{s=r+1}^{t-1} v(s)\right], t \geq t_{0} .
\end{aligned}
$$

Let $B_{1}>0$ be given. Choose $B_{2}$ such that

$$
\left|1-c\left(t_{0}-1\right)\right| M B_{1}+\alpha B_{2} \leq B_{2}
$$

We first show that $P$ maps from $S$ to $S$. By (4.5)

$$
\begin{array}{r}
|(P \varphi)(t)| \leq\left|1-c\left(t_{0}-1\right)\right| M B_{1}+\alpha B_{2} \\
\leq B_{2} \quad \text { for } t \geq t_{0}
\end{array}
$$


Thus $P$ maps from $S$ into itself. We next show that $P$ is a contraction under the supremum norm. Let $\zeta, \eta \in S$. Then

$$
\begin{aligned}
|(P \zeta)(t)-(P \eta)(t)| & \leq\left(|c(t-1)|+\sum_{r=t_{0}}^{t-1}[|b(r)-\phi(r)|]\left|\prod_{s=r+1}^{t-1} v(s)\right|\right)\|\zeta-\eta\| \\
& +\sum_{r=t_{0}}^{t-1} \mid a(r)-v\left(r|| \prod_{s=r+1}^{t-1} v(s)\|\zeta-\eta\|\right. \\
& \leq \alpha\|\zeta-\eta\| .
\end{aligned}
$$

This shows that $P$ is a contraction. Thus, by the contraction mapping principle, $P$ has a unique fixed point in $S$ which solves (4.1). Hence solutions of (4.1) are equi-bounded.

Theorem 4.8. Assume that the hypotheses of Theorem 4.7 hold. Then the zero solution of (4.1) is stable.

Proof. Let $\epsilon>0$ be given. Choose $\delta>0$ such that

$$
\left|1-c\left(t_{0}-1\right)\right| M \delta+\alpha \epsilon \leq \epsilon
$$

Let $\psi(t)$ be a bounded initial function satisfying $|\psi(t)| \leq \delta$. Define the complete metric space $S$ by

$$
S=\left\{\varphi: \mathbb{Z} \rightarrow \mathbb{R} \mid \varphi(t)=\psi(t) \text { on } \mathbb{Z}_{0} \text { and }\|\varphi\| \leq \epsilon\right\}
$$

Let $P: S \rightarrow S$ be defined by (4.4). Then, from the proof of Theorem 4.8 we have that $P$ is a contraction map and for any $\varphi \in S,\|P \varphi\| \leq \epsilon$.

Hence the zero solution of (4.1) is stable.

Theorem 4.9. Assume that the hypotheses of Theorem 4.7 hold. Also assume that

$$
\prod_{s=t_{0}}^{t-1} v(s) \rightarrow 0 \text { as } t \rightarrow \infty
$$

Then the zero solution of (4.1) is asymptotically stable.

Proof. We have already shown that the zero solution of (4.1) is stable. Let $r\left(t_{0}\right)$ be the $\delta$ of stability of the zero solution.

Let $\psi(t)$ be any initial discrete function satisfying $|\psi(t)| \leq r\left(t_{0}\right)$. Define

$$
S^{*}=\left\{\varphi: \mathbb{Z} \rightarrow \mathbb{R} \mid \varphi(t)=\psi(t) \text { on } \mathbb{Z}_{0},\|\varphi\| \leq \epsilon \text { and } \varphi(t) \rightarrow 0 \text { as } t \rightarrow \infty\right\} \text {. }
$$


Define $P: S^{*} \rightarrow S^{*}$ by (4.4). The from Theorem 4.7, the map $P$ is a contraction and it maps from $S^{*}$ into itself.

Left to show that $(P \varphi)(t) \rightarrow 0$ as $t \rightarrow \infty$.

Let $\varphi \in S^{*}$. Then the first first term on the right of (4.4) goes to zero. The second term on the right side of (4.4) goes to zero due condition (4.7) and the fact that $\varphi \in S^{*}$.

Now we show that the second term on the right side of (4.7) goes to zero as $t \rightarrow \infty$. Let $\varphi \in S^{*}$ then $|\varphi(t)| \leq \epsilon$. Also, since $\varphi(t) \rightarrow 0$ as $t \rightarrow \infty$, there exists a $t_{1}>0$ such that for $t>t_{1},|\varphi(t)|<\epsilon_{1}$ for $\epsilon_{1}>0$. Due to condition (4.7) there exists a $t_{2}>t_{1}$ such that for $t>t_{2}$ implies that

$$
\left|\prod_{s=t_{1}}^{t} v(s)\right|<\frac{\epsilon_{1}}{\alpha \epsilon} .
$$

Thus for $t>t_{2}$, we have

$$
\begin{aligned}
& \left|\sum_{r=t_{0}}^{t-1}[a(r)-v(r)] \varphi(r) \prod_{s=r+1}^{t-1} v(s)\right| \leq \sum_{r=t_{0}}^{t-1}\left|(a(r)-v(r)) \varphi(r) \prod_{s=r+1}^{t-1} v(s)\right| \\
\leq & \sum_{r=t_{0}}^{t_{1}-1}\left|(a(r)-v(r)) \varphi(r) \prod_{s=r+1}^{t-1} v(s)\right| \\
& +\sum_{r=t_{1}}^{t-1}\left|(a(r)-v(r)) \varphi(r) \prod_{s=r+1}^{t-1} v(s)\right| \\
\leq & \epsilon \sum_{r=t_{0}}^{t_{1}-1}\left|(a(r)-v(r)) \prod_{s=r+1}^{t-1} v(s)\right|+\epsilon_{1} \alpha \\
\leq & \epsilon \sum_{r=t_{0}}^{t_{1}-1}\left|[a(r)-v(r)] \prod_{s=r+1}^{t_{1}-1} v(s) \prod_{s=t_{1}}^{t-1} v(s)\right|+\epsilon_{1} \alpha \\
\leq & \epsilon\left|\prod_{s=t_{1}}^{t-1} v(s)\right| \sum_{r=t_{0}}^{t_{1}}\left|[a(r)-v(r)] \prod_{s=r+1}^{t_{1}-1} v(s)\right|+\epsilon_{1} \alpha \\
\leq & \epsilon \alpha\left|\prod_{s=t_{1}}^{t-1} v(s)\right|+\epsilon_{1} \alpha \\
\leq & \epsilon_{1}+\epsilon_{1} \alpha .
\end{aligned}
$$

This shows that the second term of (4.4) goes to zero as t goes to infinity. Showing that the last term on the right side of (4.7) goes to zero as $t \rightarrow \infty$ is similar, and hence we omit. This implies that $(P \varphi)(t) \rightarrow 0$ as $t \rightarrow \infty$.

By the contraction mapping principle, $P$ has a unique fixed point that solves $(4.1)$ and goes to zero as t goes to infinity. This concludes that the zero solution of (4.1) is asymptotically stable. 
Remark 4.10. If the delay function $g(t)$ is unbounded, then we may prove a similar theorem to Theorem 4.9 by making the additional requirement that $t-g(t) \rightarrow 0$, as $t \rightarrow \infty$.

\section{$5 \quad$ Example}

Example 5.1. Solutions of the linear neutral difference equation

$$
x\left((t+1)=\frac{2^{t+1}}{8(1+t) !} x(t-2)+\frac{2^{t+1}}{8(1+t) !} \Delta x(t-2), t \geq 0\right.
$$

are equi-bounded and the zero solution is asymptotically stable.

Proof. Let $v(t)=\frac{1}{3(1+t)}$. Comparing terms, we see that $a(t)=0, b(t)=c(t)=\frac{2^{t+1}}{8(1+t) !}$. Set $t_{0}=0$. Then (4.3) is equivalent to

$$
\begin{aligned}
|c(t-1)| & +\sum_{r=0}^{t-1}|v(r)|\left|\prod_{s=r+1}^{t-1} v(s)\right| \\
& +\sum_{r=0}^{t-1}[|b(r)-\phi(r)|]\left|\prod_{s=r+1}^{t-1} v(s)\right| \\
& \leq \frac{2^{t}}{8(t) !}+\sum_{r=0}^{t-1} \prod_{s=r}^{t-1} \frac{1}{3(1+s)}+\sum_{r=0}^{t-1} \frac{2^{r}}{8(1+r) !} \prod_{s=r+1}^{t-1} \frac{1}{3(1+s)}
\end{aligned}
$$

Now,

$$
\begin{aligned}
\sum_{r=0}^{t-1} \frac{2^{r}}{8(1+r) !} \prod_{s=r+1}^{t-1} \frac{1}{3(1+s)} & \leq 1 / 3 \sum_{r=0}^{t-1} \frac{2^{r}}{8(1+r) !} \frac{1}{(r+2)(r+3) \ldots(t)} \\
& \leq 1 / 3 \sum_{r=0}^{t-1} \frac{2^{r}}{8 t !} \\
& \leq \frac{1}{24 t !}\left(2^{t}-1\right) \leq \frac{2^{t}}{24 t !} .
\end{aligned}
$$

Similarly, by estimating $\frac{1}{1+s} \leq 1$, for $s \geq 0$, we have that

$$
\begin{aligned}
\sum_{r=0}^{t-1} \prod_{s=r}^{t-1} \frac{1}{3(1+s)} & \leq \sum_{r=0}^{t-1}\left(\frac{1}{3}\right)^{t-r} \\
& \leq\left(\frac{1}{3}\right)^{t} \sum_{r=0}^{t-1} 3^{r}=\left.\left(\frac{1}{3}\right)^{t}\left[\frac{3^{r}}{2}\right]\right|_{0} ^{t-1} \\
& \leq \frac{1}{6}\left[1-2^{1-t}\right] \leq 1 / 6
\end{aligned}
$$


Combining the two inequalities we end up with

$$
\begin{aligned}
|c(t-1)| & +\sum_{r=0}^{t-1}|v(r)|\left|\prod_{s=r+1}^{t-1} v(s)\right| \\
& +\sum_{r=0}^{t-1}[|b(r)-\phi(r)|]\left|\prod_{s=r+1}^{t-1} v(s)\right| \\
& \leq \frac{2^{t}}{8(t) !}+\frac{1}{3}+\frac{2^{t}}{24 t !} \\
& \leq \frac{1}{4}+\frac{1}{6}+\frac{1}{12}=\frac{1}{2}<1 .
\end{aligned}
$$

Hence (4.3) is satisfied. It is clear that condition (4.7) is satisfied for the specified value of $v$. This implies the zero solution is asymptotically stable, by Theorem 4.9. Left to show solutions are equi-bounded.

Since $t_{0}=0$, we have that $\mathbb{Z}_{0}=[-2,0]$.

Let $B_{1}>0$ be given and $\psi(t): \mathbb{Z}_{0} \rightarrow \mathbb{R}$ be a given initial function with $|\psi(t)| \leq B_{1}$. We need to choose $B_{2}$ so that (4.5) is satisfied. It is clear that $c\left(t_{0}-1\right)=c(-1)=\frac{1}{8}$, and hence $\left|1-c\left(t_{0}-1\right)\right|=1-\frac{1}{8}=\frac{7}{8}$. In addition

$$
\left|\prod_{s=0}^{t-1} v(s)\right| \leq M
$$

is satisfied for $M=\frac{1}{3}$. From the above calculation for asymptotic stability, we see that $\alpha=\frac{1}{2}$. Now we choose $B_{2}$ such that

$$
\frac{7}{24} B_{1} \leq \frac{B_{2}}{2}
$$

Then, in our case, inequality (4.5) corresponds to

$$
\left|1-c\left(t_{0}-1\right)\right| M B_{1}+\alpha B_{2} \leq B_{2} .
$$

Or equivalently,

$$
\frac{7}{24} B_{1}+\frac{B_{2}}{2} \leq B_{2}
$$

is satisfied.

Remark 5.2. We mention that the work of Islam-Yankson in [12] can not be applied to our example due to the absence of the linear term $a(t) x(t)$.

It is worth mentioning that the results of Section 4 can be easily extended to the nonlinear neutral difference equation

$$
x(t+1)=a(t) x(t)+c(t) \Delta x(t-g(t))+q(t, x(t), x(t-g(t)))
$$


where $a(t), c(t)$ and $g(t)$ are defined as before. We assume that, $q(t, 0,0)=0$ for the stability and $q$ is locally Lipschitz in $x$ and $y$. That is, there is a $K>0$ so that if $|x|,|y|,|z|$ and $|w| \leq K$ then

$$
|q(x, y)-q(z, w)| \leq L|x-z|+E|y-w|
$$

for some positive constants $L$ and $E$.

Note that

$$
\begin{aligned}
|q(x, y)| & =|q(x, y)-q(0,0)+q(0,0)| \\
& \leq|q(x, y)-q(0,0)|+|q(0,0)| \\
& \leq L|x|+E|y| .
\end{aligned}
$$

Remark 5.3. The method of Section 4 can be easily used to extend the existence of periodic solutions to systems of the form of (4.1) and (5.2), see [15]. 


\section{References}

[1] Adivar, M,. Islam, M. and Raffoul, Y., Separate contraction and existence of periodic solutions in totally nonlinear delay differential equations, Hacettepe Journal of Mathematics and Statistics, 41 (1) (2012), 1-13.

[2] T. Burton, Volterra Integral and Differential Equations, Academic Press, New York, 1983.

[3] T. Burton, Stability and Periodic Solutions of Ordinary and Functional Differential Equations, Academic Press, New York, 1985.

[4] T. Burton and T. Furumochi, Fixed points and problems in stability theory, Dynam. Systems Appl. 10(2001), 89-116.

[5] Burton, T.A. Integral equations, implicit functions, and fixed points, Proc. Amer. Math. Soc. 124 (1996), 2383-2390.

[6] S. Elaydi, An Introduction to Difference Equations, Springer, New York, 1999.

[7] S. Elaydi, Periodicity and stability of linear Volterra difference systems, J. Math. Anal. Appl. ,181(1994), 483-492.

[8] S. Elaydi and S. Murakami, Uniform asymptotic stability in linear Volterra difference equations, J. Differ. Equations Appl., 3(1998), 203-218.

[9] P. Eloe, M. Islam and Y. Raffoul, Uniform asymptotic stability in nonlinear Volterra discrete systems, Special Issue on Advances in Difference Equations IV, Computers Math. Appl., 45(2003), 1033-1039.

[10] Y. Hino and S. Murakami, Total stability and uniform asymptotic stability for linear Volterra equations, J. London Math. Soc., 43(1991), 305-312.

[11] M. Islam and Y. Raffoul, Exponential stability in nonlinear difference equations, J. Differ. Equations Appl., 9(2003), 819-825.

[12] M. Islam and E. Yankson Boundedness and stability in nonlinear delay difference equations employing fixed point theory, Electronic Journal of Qualitative Theory of Differential Equations 2005, No. 26, 1-18.

[13] W. Kelley and A. Peterson, Difference Equations: An Introduction with Applications, Harcourt Academic Press, San Diego, 2001.

[14] J. Liu, A First Course In The Qualitative Theory of Differential Equations,Pearson Education, Inc., Upper Saddle River, New Jersey 07458, 2003. 
[15] M. Maroun and Y. Raffoul, Periodic solutions in nonlinear neutral difference equations with functional delay, J. Korean Math. Soc. 42 (2005), no. 2, 255-268.

[16] R. Medina, Asymptotic behavior of Volterra difference equations, Computers Math. Appl. 41(2001), 679-687.

[17] R. Medina, The asymptotic behavior of the solutions of a Volterra difference equation, Computers Math. Appl. 181(1994), 19-26.

[18] Y. Raffoul, Qualitative Theory of Volterra Difference Equations, Springer, New York, 2018.

[19] Y. Raffoul, Stability and periodicity in discrete delay equations, J. Math. Anal. Appl. 324 (2006) 1356-1362.

[20] Y. Raffoul, Stability in neutral nonlinear differential equations with functional delays using fixed point theory, Mathematical and Computer Modelling, 40(2004), 691-700.

[21] Y. Raffoul, General theorems for stability and boundedness for nonlinear functional discrete systems, J. Math. Anal. Appl. ,279(2003), 639-650. 